\title{
Transferência de tecnologia para a cultura da soja - a experiência da COPACOL
}

\section{Technology transference in soybean culture-COPACOL experience}

\author{
Lineu Alberto Domit ${ }^{*}$; Milton Dalbosco ${ }^{2}$; \\ Rubem Marco de Salles Santos ${ }^{2}$; Maria de Fátima Guimarães ${ }^{3}$
}

\begin{abstract}
Resumo
Este trabalho analisou a metodologia Sistema de Treino e Visita (T\&V) aplicada pela Cooperativa Agroindustrial Consolata-COPACOL na transferência de tecnologia, tendo como objetivo mostrar que um processo de transferência de tecnologia, quando desenvolvido de forma contínua e sistêmica, pode dinamizar a chegada e adoção dessas tecnologias ao produtor rural com resultados positivos para todos os participantes do sistema. A análise focou o Sistema T\&V, que é uma metodologia já utilizada pela EMBRAPA Soja, unidade da Empresa Brasileira de Pesquisa Agropecuária - EMBRAPA, em parceria com o Instituto Paranaense de Assistência Técnica e Extensão Rural - EMATER, Instituto Agronômico do Paraná - IAPAR e Organização da Cooperativas do Paraná - OCEPAR. Concluiu-se que o Sistema de $T \& V$ pode ser uma ferramenta importante para o aprimoramento do processo de validação e de transferência das tecnologias geradas pela pesquisa. Na COPACOL, o T\&V possibilitou a evolução tecnológica dos técnicos e produtores participantes, o aumento da renda obtida pelos cooperados participantes do $\mathrm{T} \& \mathrm{~V}$ quando comparada com a média obtida por todos os cooperados e a diminuição do tempo para a adoção de novas tecnologias.
\end{abstract}

Palavras-chave: Treino e visita; T\&V; cooperativa

\begin{abstract}
This paper analyzed the technology transfer methodology, the Training and Visit System (T\&V), applied by Agroindustrial Cooperative of Consolata - COPACOL. The objective was to demonstrate that the continuous and holistic application of a technology transfer system may speed up the awareness and the adoption of these technologies by the farmers and result in positive outcomes for all actors and institutions involved in the process. The methodology of technology transfer analyzed, the T\&V System, is already used by Embrapa Soybean, a branch of Brazilian Agricultural Research Corporation - EMBRAPA, Rural Extension and Technical Assistance Corporation - EMATER, Agronomic institute of Parana IAPAR and Cooperative Organization of Parana - OCEPAR as partners. It was concluded that the T\&V System may be an important tool for improvement of the validation and transfer of technologies generated by research institutions. In the COPACOL, the T\&V contributed with the technological development of the technicians and farmers. It contributed also, with the growth of the profit obtained by the farmers who participated of the T\&V as compared with the average growth obtained by the other farmers, members of the cooperative. Finally, it was observed that the time of adoption of new technologies was decreased with the application of the T\&V System.
\end{abstract}

Key words: Training and visit. T\&V. Cooperative.

1 Engenheiro Agrônomo, Doutor. Empresa Brasileira de Pesquisa Agropecuária - Centro Nacional de Pesquisa de Soja em Londrina/ PR.E-mail: domit@cnpso.embrapa.br

2 Engenheiro Agrônomo. Cooperativa Agroindustrial Consolata-COPACOL em Cafelândia/PR.

3 Engenheira Agrônoma, Doutora. Universidade Estadual de Londrina em Londrina/PR

* Autor para correspondência

Recebido para publicação 25/01/07 Aprovado em 04/12/07 


\section{Introdução}

O aumento da renda dos produtores rurais, principalmente os participantes do segmento da agricultura familiar, é dependente da implantação de práticas técnico-gerenciais que possibilitem uma melhor organização e aproveitamento da atividade produtiva. Estas práticas devem levar à diminuição de custos e/ou aumento da produtividade, agregar valores à produção e colaborar para a preservação e melhoria do ambiente produtivo.

Nas regiões produtoras de soja, milho e trigo dos Estados do Paraná, Santa Catarina e São Paulo predominam pequenos e médios produtores, que necessitam de assistência técnica efetiva que possibilite o acesso a informações e tecnologias que resultem em melhoria de renda e qualidade de vida. Nessas regiões, há disponibilidade de agentes de Assistência Técnica e Extensão Rural (ATER), entretanto, para uma assistência mais eficaz, há necessidade de atualização técnica permanente. $\mathrm{O}$ sistema de Treino \& Visita (T\&V), por meio de um fórum permanente de discussão entre pesquisa, ATER e produtores rurais, pode aprimorar o processo de transferência de tecnologias, possibilitando a chegada mais rápida das tecnologias aos agricultores participantes, bem como obter "feedback" sobre as tecnologias indicadas para os agentes de pesquisa.

\section{Transferência de Tecnologia}

Diversos documentos, trabalhos e teses citam problemas e dificuldades no processo de transferência tecnológica. Alves (2001) em estudo sobre a transferência de tecnologia na EMBRAPA, afirma que, desde 1973, poucos avanços ocorreram, principalmente no processo de validação das tecnologias junto aos agricultores e que boa parte das tecnologias desenvolvidas permanecem estocadas nas instituições de pesquisa. Também Duarte (2004) analisa que a EMBRAPA, além do problema de concepção do sistema de difusão, que não oferece um conjunto de procedimentos estratégicos para transferência de tecnologias, nem possui política consolidada sobre o assunto, enfrentou ao longo de sua história restritas condições de atuação dos setores responsáveis pela ligação com a assistência técnica e extensão rural, os agricultores e outros usuários da tecnologia.

Já o Programa de Fortalecimento e Modernização dos Processos de Transferência Tecnológica da EMBRAPA concluiu que os dias atuais estão exigindo novos procedimentos de transferência de tecnologia. As demandas por tecnologias têm crescido rapidamente, pois, as políticas econômicas globalizadas exigem mais eficiência das cadeias produtivas que formam o setor agrícola. A concorrência aumentou e é preciso produzir a custos menores, com maior qualidade e oportunidade. Com isso acontecendo rapidamente, os sistemas que disponibilizam as tecnologias têm que ser, necessariamente, muito mais ágeis (EMBRAPA, sd.). Propostas para aprimorar o processo de transferência de tecnologia já existem e, em sua maioria, citam a necessidade de considerar também a realidade regional/local com envolvimento de setores públicos e privados que, de forma integrada com os agricultores, desenvolvem todas as etapas do processo de transferência tecnológica (LEMOS, 2000 e DUARTE, 2004). Também é fundamental o envolvimento das instituições de P\&D na interação com técnicos e produtores, possibilitando o fluxo da informação nos dois sentidos (GUIMARÃES, 2000).

\section{Sistema Treino \& Visita}

O Banco Mundial, objetivando aprimorar o processo de transferência de tecnologia nos países subdesenvolvidos, principalmente da África e da Ásia, divulgou e financiou, um método de difusão desenvolvido por Benor, Harrison e Baxter (1984) denominado "T\&V", sistema de treino e visita. Segundo os autores, este sistema visa fortalecer os elos de ligação entre a pesquisa agropecuária, a assistência técnica e extensão rural e os produtores rurais, criando um fluxo sistemático de informações. 
O seu funcionamento ocorre pela formação e treinamento de especialistas na extensão rural que, em constante contato com a pesquisa, formam e treinam monitores no meio rural para repassar a informação aos produtores. Os produtores participantes serão, por sua vez, os disseminadores das idéias nos seus sistemas sociais através do efeito visual e demonstrativo que exercerão.

No Estado do Paraná, segundo Domit e Adegas (1999), foi estabelecida, em 1996, uma parceria entre a EMBRAPA Soja, EMATER-PR, IAPAR e agentes da ATER oficial e privada, para o desenvolvimento de um projeto, utilizando a metodologia do $\mathrm{T} \& \mathrm{~V}$ com adaptações para a realidade brasileira, com o objetivo de aprimorar o processo de validação e de transferência das tecnologias desenvolvidos pelas instituições de pesquisa. Segundo os autores, este trabalho possibilitou a capacitação contínua dos técnicos sobre a cultura de soja, milho e de trigo, a criação de um fluxo sistemático e organizado de repasse destas informações até o agricultor, o aprimoramento do relacionamento técnico-gerencial de todos os participantes (pesquisa, agentes da ATER e agricultor) e da capacidade gerencial dos produtores envolvidos. A experiência bem sucedida no Estado do Paraná possibilitou uma aproximação bastante profícua entre a EMBRAPA e demais instituições parceiras, tanto da esfera pública quanto da esfera privada, permitindo a tão desejável redução do tempo entre a disponibilização do resultado da pesquisa e sua adoção, conforme apontam Vieira, Oliveira e Domit (2004) que mostram o aumento da produtividade da cultura do girassol na região CentroOeste do Brasil em decorrência da adoção do T\&V. Estas avaliações empíricas ratificam o sucesso do $\mathrm{T} \& \mathrm{~V}$, além de reforçar o seu caráter inovador como um sistema de transferência de tecnologia, que tem permitido, à EMBRAPA e às instituições parceiras, a promoção do desenvolvimento em seu conceito mais amplo, incluindo as perspectivas tecnológica, ambiental, econômica, social e cultural. Por meio da adoção do Sistema de T\&V, a EMBRAPA, juntamente com seus parceiros, tem tido a oportunidade de acompanhar o comportamento das tecnologias por eles geradas e promovidas e, a partir daí, retroalimentar o processo de pesquisa e desenvolvimento (P\&D). Além disso, Vieira, Oliveira e Domit (2004) ressaltam a contribuição do T\&V na formação de uma rede de validação de tecnologias, que permite melhor adaptação dos resultados da pesquisa para diferentes regiões do país.

\section{Validação do T\&V: a Experiência da Embrapa}

Relatórios internos sobre as atividades desenvolvidas pela equipe de TT da EMBRAPA Soja indicam que, em parceria com a EMATER-PR, o T\&V Soja iniciou na safra 1996/97 e, em seguida, foram incluídos na parceria o IAPAR e a Ocepar/ Sescoop-PR. A partir desta rede de parcerias, o projeto passou a ser denominado $\mathrm{T} \& \mathrm{~V}$ Grãos. O sistema funcionou por meio da capacitação contínua de um grupo de agentes de assistência técnica (oficial e privada) - Os técnicos multiplicadores I (TM I) que estavam em contato direto e constante com pesquisadores e outros especialistas, sendo informados das tecnologias disponibilizadas pelas instituições de pesquisa quase em tempo real. Os TM I transferiram tais tecnologias aos técnicos de campo - multiplicadores II (TM II) - que desempenharam a função de multiplicadores e tinham o compromisso de repassar as tecnologias a grupos organizados de agricultores, além de assessorarem aqueles que queriam adotar tais tecnologias em suas propriedades.

A Tabela 1 mostra os TM I participantes do T\&V Soja/Grãos no período de 1996/97 a 2003/04. 
Tabela 1. Númerode reuniões do comitê do T\&V Soja/Grãos realizadas no período de 1996 a 2004 e número de TM I participantes.

\begin{tabular}{ccc}
\hline Período & Reuniões realizadas & TM I \\
$1996 / 97$ & 04 & 8 \\
$1997 / 98$ & 06 & 16 \\
$1998 / 99$ & 06 & 16 \\
$1999 / 00$ & 07 & 18 \\
$2000 / 01$ & 6 & 22 \\
$2001 / 02$ & 12 & 35 \\
$2002 / 03$ & 8 & 68 \\
$2003 / 04$ & & 45 \\
\hline
\end{tabular}

Fonte: Embrapa Soja

Deve-se considerar que cada TM I transfere as informações recebidas para, em média, 10 TM II que, por sua vez, repassam para no mínimo 10 agricultores, resultando em um grande número de produtores rurais recebendo e validando regionalmente as tecnologias de forma direta e eficiente. Neste período, os principais resultados observados foram a capacitação e reciclagem permanente de todos os participantes, que possibilitou a evolução técnica-gerencial-profissional, principalmente dos TM I, que se tornaram referência técnica na região; a formação de uma estrutura sistêmica e permanente, que resultou numa forte integração entre os participantes, numa rede regional/ local de validação de tecnologias com discussão e difusão de casos de sucesso, no desenvolvimento de projetos pilotos (Ex. Manejo da Fertilidade do Solo/ COPACOL) e num maior efeito de multiplicação e de retroalimentação para a pesquisa; a criação de um sistema dinâmico de comunicação entre os pesquisadores e técnicos (e-mail, internet) e a participação na melhoria da renda dos produtores e do ambiente produtivo.

\section{A COPACOL e sua Atuação}

A Cooperativa Agroindustrial Consolata COPACOL foi fundada em 1963 e em 2003, contava com a participação de 4.263 cooperados, sendo na grande maioria micro e pequenos produtores. A cooperativa atua diretamente em seis municípios da região oeste do Paraná: Cafelândia, Nova Aurora, Formosa do Oeste, Jesuítas, Tupãssi e Iracema do Oeste, totalizando uma área cultivada de $100 \mathrm{mil}$ hectares, onde as principais culturas plantadas são soja, milho, trigo, café e algodão. Sua principal atividade, atualmente, é a avicultura de corte. Relatórios internos e informações obtidas junto à diretoria e equipe técnica da COPACOL indicam que a agricultura da região foi iniciada na década de 60 , respaldada unicamente nas informações e técnicas trazidas pelo conhecimento "hereditário". No início da década de 70, a mecanização agrícola teve participação direta na ampliação nas áreas de plantio, e, com a mecanização, surgiram os problemas de conservação de solo e de manejo das culturas, o que levou a uma crescente demanda de informações técnicas. Em 1975, foi criado o Departamento Técnico da COPACOL. Na década de 80, a Cooperativa dispunha de eficiente departamento técnico, com número de profissionais suficiente para atender o seu quadro social. Porém, isto não resultava em evolução tecnológica da região. O grande número de tecnologias geradas pelas instituições de pesquisa tinha baixa adoção pelos produtores e uma defasagem de vários anos, o que caracterizava uma falha no 
sistema de transferência de tecnologia. A partir de 1996, objetivando aprimorar o seu processo de assistência técnica e de transferência de tecnologia, a COPACOL estabeleceu uma parceria com a EMBRAPA Soja e o IAPAR, passando a integrar o sistema $\mathrm{T} \& \mathrm{~V}$.

\section{Material e Métodos}

As atividades de assistência técnica estavam fundamentadas em visitas exclusivas, solicitadas pelos Associados que popularmente denominava-se de "Assistência Bombeiro". Os profissionais tinham a incumbência mecânica de fazer laudos e atender somente às solicitações dos Associados. Neste período, havia grande predominância de Técnicos Agrícolas e poucos Engenheiros Agrônomos e existia uma heterogeneidade na capacidade técnica desses profissionais. Estes profissionais eram oriundos de diversos centros educacionais, com formação acadêmica e informações técnicas das mais diversas e, muitas vezes, não adaptadas à região de atuação. Tudo isso levou à falta de padronização nas indicações técnicas e, conseqüentemente, a resultados muitas vezes não desejáveis como a assistência técnica por solicitação (bombeiro) e a submissão à forte pressão das revendedoras de insumos. Como conseqüência, na prática, observou-se que poucas tecnologias eram efetivamente adotadas, tendo poucos produtores com resultados satisfatórios e muitos com resultados que não permitiam a própria sustentabilidade. A reciclagem dos profissionais era realizada por meio de palestras pontuais, muitas delas ministradas por representantes das empresas multinacionais de insumos, cujo objetivo principal era capacitar a equipe técnica da COPACOL para a venda do seu portifólio de produtos. Com isso, a imagem dos profissionais para os Associados era de "veneneiros", ou seja, só sabiam recomendar agroquímicos. O contato mais direto com a pesquisa era esporádico e existiam dificuldades de uma integração mais ampla com as instituições de pesquisa. Em 1996, a COPACOL realizou um levantamento junto ao quadro de associados que indicou que $88 \%$ dos associados possuíam menos de 50 hectares; que a maioria absoluta tinha pouca escolaridade; que as famílias apresentavam grande número de netos em idade escolar e filhos em idade de assumir a sua independência financeira, muitas vezes levando à divisão das propriedades, e que estas, apesar da boa aptidão agrícola de suas terras e da disponibilidade de tecnologias, apresentavam baixos níveis de produtividade e rentabilidade. Baseada nesta realidade, a Cooperativa entendeu que o seu método de assistência técnica não atingia seus associados e não utilizava as potencialidades da região. Isto, inevitavelmente, iria redundar em êxodo rural, pela inviabilização técnica e econômica das pequenas propriedades e, seguramente, comprometeria o desenvolvimento da Cooperativa e da região. Visando reverter essa situação, ainda em 1996, a COPACOL estabeleceu uma parceria com a EMBRAPA Soja e IAPAR com o objetivo de aprimorar o seu processo de assistência técnica e de transferência de tecnologia, ingressando no Sistema de T\&V para as culturas de soja, milho, trigo e outras culturas que participam do sistema de produção de grãos. O T\&V COPACOL foi iniciado na safra 1996/97 e sua implantação e desenvolvimento foram compostos pelas seguintes etapas:

Etapa 1 - Convite e motivação: a cooperativa foi contatada por um representante da EMBRAPA Soja que informou sobre o $T \& V$ e convidou a COPACOL para reunião ampliada, onde seria exposta e discutida a proposta. Em seguida, a diretoria e a equipe técnica decidiram implantar e priorizar este sistema de TT. Na Cooperativa, o T\&V foi denominado de PPPR Programa de Profissionalização do Produtor Rural.

Etapa 2 - Implantação e desenvolvimento do $\mathrm{T} \& \mathrm{~V}$ : o processo de $\mathrm{T} \& \mathrm{~V}$ aplicado pela EMBRAPA Soja e seus parceiros foi baseado no fluxograma apresentado na Figura 1. 


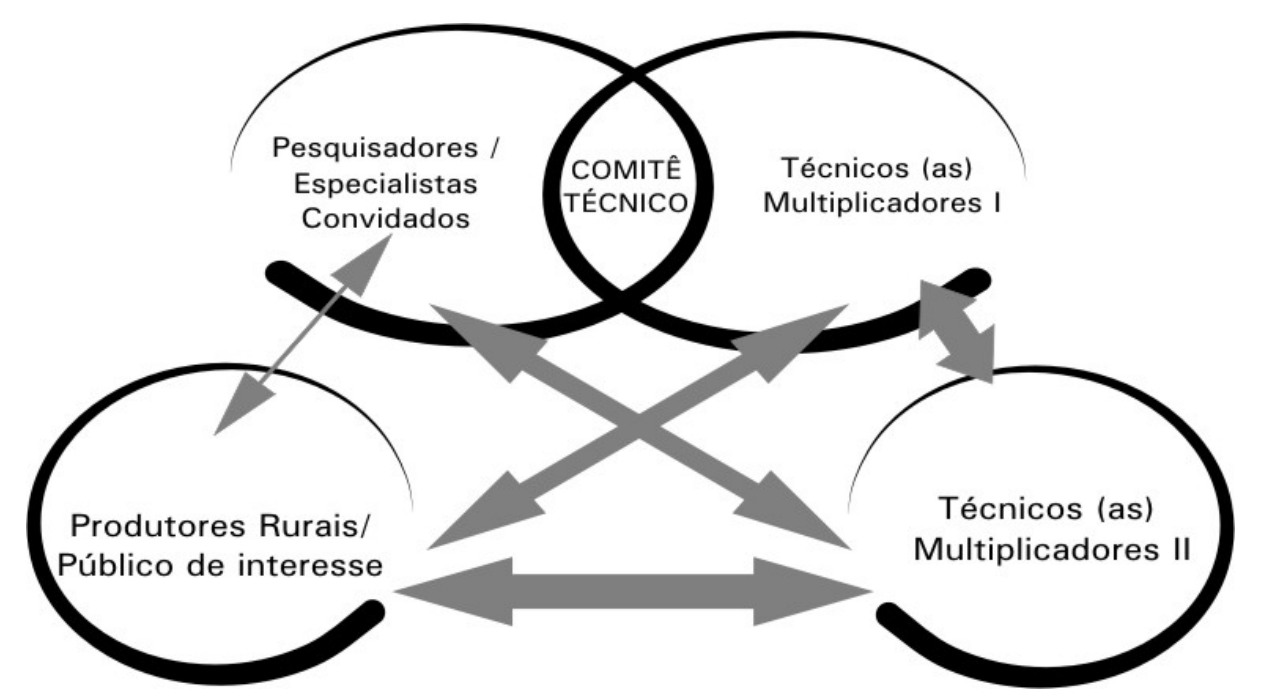

Figura 1. Fluxograma do esquema operacional aplicado no processo T\&V. Fonte: Domit et al. (2007).

A equipe de trabalho foi composta por um comitê técnico (CT), um coordenador geral do processo, um grupo de agentes do "sistema pesquisa" e um grupo de agentes do "sistema ATER". Os agentes do "sistema pesquisa" são profissionais e pesquisadores, com reconhecida competência técnica, integrantes dos quadros da EMBRAPA, das Instituições de Pesquisa Estaduais (IAPAR), das Instituições de Ensino Superior e de outras instituições que possam contribuir para o processo. Os agentes do "sistema ATER" são os TM I, que coordenam e repassam os conhecimentos e tecnologias para os TM II, que, por sua vez, coordenam e repassam os conhecimentos e tecnologias para os produtores. A função dos agentes do sistema de pesquisa é de apoiar os TM I, através de um processo de integração de ações, numa relação profissional não hierárquica, mas de troca, com o intuito de atender as demandas e potencializar a transferência das soluções que serão repassadas para os TM II e, em seguida, para os produtores. No Comitê Técnico, a interface do sistema pesquisa com o sistema de ATER, é que se estabelece a coordenação da gestão do conhecimento. Os atores possuem graus de relacionamento diferenciados, conforme a intensidade das setas componentes da figura abaixo. Para o T\&V aplicado na COPACOL, foi selecionado um engenheiro agrônomo para exercer a função de Multiplicador I para as culturas da Soja,
Milho e Trigo. Este Multiplicador participou das reuniões do Comitê Técnico (CT) organizado pelas Instituições de Pesquisas, com o objetivo de discutir as tecnologias e conhecimentos indicados pela pesquisa buscando a viabilização técnica e econômica das propriedades, por meio dos processos produtivos destas culturas. O TM I tinha a tarefa de repassar para os TM II, de forma organizada, o conhecimento adquirido no CT e também era o responsável pela Estação Experimental, onde as tecnologias geradas pela Pesquisa foram validadas para a região e, depois disso, recomendadas pela equipe técnica. Cada TM II (assessor técnico de campo) ficou responsável por uma área de atuação e definiu o seu grupo de associados participantes do PPPR (a participação no programa é por livre adesão). Todas as inovações tecnológicas foram (e continuam sendo) repassadas para os grupos, bem como os assuntos técnicos, econômicos ou outros que foram demandados. O grupo também sugeriu os cursos e palestras necessárias para o período e o Multiplicador II elaborou a agenda e tomou as providências para a sua realização. As informações técnicas são repassadas aos associados e, posteriormente, são realizadas reuniões de avaliação dos procedimentos e resultados alcançados, inclusive com análises de custos. O Multiplicador II tinha também a responsabilidade de demonstrar para o 
associado o seu crescimento econômico em função da sua atuação com a Cooperativa, seja pela participação dos seus negócios, pelo aumento de sua produtividade ou mesmo pela entrega da produção e aquisição de insumos.

Etapa 3 - Avaliação e divulgação: Em cada safra agrícola foi levantada a produtividade e o custo variável da soja obtida por cada produtor do $\mathrm{T} \& \mathrm{~V}$. Estes dados foram comparados com os obtidos pelo total de cooperados. Também foram registradas outras informações que poderiam indicar a evolução dos cooperados e da Cooperativa. A divulgação foi realizada por intermédio de relatório anual que foi apresentado e discutido na COPACOL e também no Comitê Técnico do T\&V Grãos.

Todas as informações que foram utilizadas para analisar a experiência da COPACOL com a implantação e desenvolvimento do $\mathrm{T} \& \mathrm{~V}$ foram obtidas por meio dos relatórios anuais elaborados pelos TM I, de documentos internos da Cooperativa e também por intermédio de contatos pessoais com a diretoria e equipe técnica.

\section{Resultados e Discussão}

O T\&V COPACOL possibilitou maior aproximação da equipe técnica da cooperativa com as instituições de pesquisa e significativa evolução técnica dos profissionais de campo, o que levou a um processo de transferência de tecnologia mais eficiente. Este processo favoreceu a retroalimentação de informações para os pesquisadores, o aprimoramento do processo de validação regional das mais recentes tecnologias geradas pela pesquisa e o repasse para toda a equipe técnica. Desta forma, a COPACOL deixou de prestar assistência técnica para resolver problemas individuais de produtores $\mathrm{e}$ passou a realizar assessoria técnica de forma integrada e contínua para grupos de produtores. $\mathrm{O}$ $\mathrm{T} \& \mathrm{~V}$ também possibilitou a diminuição do tempo para adoção, pelos produtores, de novas recomendações da pesquisa. Estes resultados estão diretamente relacionados com os objetivos do sistema $\mathrm{T} \& \mathrm{~V}$ desenvolvido por Benor et al. (1984), com os resultados obtidos por Domit e Adegas (1999) com o desenvolvimento do T\&V Grãos nos Estados do Paraná, São Paulo e Santa Catarina e também com os de Vieira, Oliveira e Domit (2004) com a cultura do girassol no Estado de Goiás.

Algumas dificuldades existentes no processo de TT são relatadas por Alves (2001), que afirma que o processo de TT da EMBRAPA possibilitou poucos avanços na validação de tecnologias e que muitas tecnologias permaneceram estocadas nas Unidades de Pesquisa e, por Duarte (2004), que cita que, na EMBRAPA, faltam: uma política de TT, procedimentos estratégicos e condições que favoreçam o desenvolvimento de atividades de TT. Alguns autores sugeriram modificações no processo TT, como: o documento (EMBRAPA, sd.) que cita que os sistemas de TT têm que ser mais ágeis, os estudos de Lemos (2000) e Duarte, (2004) que indicam que os processos de TT devem considerar a realidade local/regional e integrar todos os autores e Guimarães (2000), que sugere que o envolvimento de P\&D com técnicos e produtores possibilita o fluxo de informações nos dois sentidos.

$\mathrm{O}$ caso T\&V COPACOL demonstrou que um processo sistêmico e contínuo de TT foi um aprimoramento na TT tradicional, principalmente a desenvolvida pela maioria das Unidades de Pesquisa da EMBRAPA e que, executado desta forma, apresenta bons resultados.

Na Tabela 2 são apresentados um conjunto de indicadores (estruturais, resultados técnicos e econômicos) que foram utilizados para comparar o T\&V COPACOL com o total da Cooperativa no período de 1996/97 a 2003/04. Observa-se que na COPACOL, de maneira geral, os indicadores são bastante estáveis durante o período analisado, com exceção do número de cooperados, que diminuiu com o decorrer dos anos e dos custos de produção da soja e do milho, que aumentaram a partir de 1999/00 e que tiveram um aumento maior ainda a partir de 2002/03. 
Tabela 2. Estrutura e resultados médios obtidos pelo total de produtores da Copacol e pelos produtores participantes do PPPR - T\&V Copacol no período de 1996/97 a 2003/04.

\begin{tabular}{|c|c|c|c|c|c|c|c|c|c|}
\hline \multirow[t]{2}{*}{ Indicadores de evolução } & \multicolumn{9}{|c|}{ Safras } \\
\hline & $96 / 97$ & $97 / 98$ & $98 / 99$ & $99 / 00$ & $00 / 01$ & $01 / 02$ & $02 / 03$ & $03 / 04$ & Média \\
\hline Técnicos & 34 & 34 & 34 & 35 & 36 & 38 & 40 & 40 & \\
\hline Produtores & 4.892 & 4.823 & 4.470 & 4.385 & 4.332 & 4.184 & 4.247 & 4.263 & \\
\hline Soja (ha) & 74600 & 76600 & 70800 & 70200 & 65000 & 73640 & 76400 & 86908 & \\
\hline Milho (ha) & 7000 & 6771 & 7145 & 7750 & 16150 & 8400 & 8810 & 7.435 & \\
\hline Soja (sc/ha) & 43,3 & 45,4 & 44,6 & 40,9 & 40,9 & 50,0 & 48,0 & 51,0 & 45,5 \\
\hline Custo Soja (R\$/ha) & - & 325,5 & 327,7 & 431,7 & 438,1 & 452,0 & 827,3 & 908,7 & 530,1 \\
\hline Milho (sc/ha) & - & - & - & 108,7 & 128,9 & 121,1 & 145,0 & 134,0 & 127,5 \\
\hline Custo Milho (R \$/ha) & - & - & - & 570,5 & 621,4 & 611,8 & 1137,0 & 1420,0 & 872,1 \\
\hline Téc Multiplicador I & 01 & 01 & 01 & 01 & 01 & 01 & 01 & 01 & \\
\hline Téc Multiplicador II & 21 & 22 & 23 & 23 & 24 & 32 & 35 & 35 & \\
\hline Municípios & 07 & 07 & 07 & 07 & 07 & 08 & 08 & 08 & \\
\hline Produtores & 316 & 329 & 337 & $\begin{array}{c}332 \\
(278)^{*}\end{array}$ & $\begin{array}{c}347 \\
(284)\end{array}$ & $\begin{array}{c}1440 \\
(1224)\end{array}$ & $\begin{array}{c}1440 \\
(1224)\end{array}$ & $\begin{array}{c}1.442 \\
(1240)\end{array}$ & \\
\hline Soja (ha) & 9500 & 9815 & 10047 & 10047 & 8994 & 43600 & 45058 & 44270 & \\
\hline Milho (ha) & - & - & - & 1655 & 3044 & 6800 & 5342 & 6147 & \\
\hline Soja (sc/ha) & 47,5 & 50,8 & 50,0 & 42,0 & 58,7 & 53,7 & 57,4 & 53,5 & 51,7 \\
\hline Custo Soja (R\$/ha) & - & 318,9 & 327,2 & 431,7 & 451,6 & 431,7 & 827,3 & 904,3 & 527,5 \\
\hline Milho(sc/ha) & - & - & - & 127,3 & 151,2 & 135,1 & 145,8 & 144,0 & 140,7 \\
\hline Custo Milho (R\$/ha) & - & - & - & 591,3 & 621,5 & 591,3 & 1137,0 & 1401,0 & 868,4 \\
\hline
\end{tabular}

Fonte: COPACOL

( ) * Agricultores familiares: Propriedade com no máximo 3 módulos rurais, sem empregados registrados e tendo como atividade principal a Agricultura

No T\&V, os indicadores se mostram semelhantes aos da COPACOL, sendo diferente na evolução no número de TM II e de produtores participantes do $\mathrm{T} \& \mathrm{~V}$. Isso demonstra que o $\mathrm{T} \& \mathrm{~V}$ passou por um processo de validação até a safra 2000/01 e, depois, foi adotado por um número maior de técnicos e produtores. Ressalta-se que a participação dos produtores não era obrigatória. A mesma tabela mostra que a média de produtividade nos produtores do $\mathrm{T} \& \mathrm{~V}$ foi superior em $6 \mathrm{sc} / \mathrm{ha}$ na soja e $13 \mathrm{sc} / \mathrm{ha}$ no milho, sendo o custo variável bastante semelhante para os dois grupos de produtores e indicando que o processo de TT adotado foi importante para o aumento da renda (margem bruta) dos produtores participantes do T\&V. Vieira, Oliveira e Domit (2004) também observaram aumento de produtividade na cultura do girassol na região Centro-Oeste do Brasil em decorrência da aplicação do T\&V. 


\section{Conclusão}

Baseado no estudo da experiência da COPACOL com a implantação e desenvolvimento do sistema $\mathrm{T} \& \mathrm{~V}$ no processo de TT para a cultura da soja no período de 1996 a 2004, conclui-se que:

1. A metodologia do $T \& V$ possibilitou uma maior integração da EMBRAPA Soja com a equipe técnica da Cooperativa, resultando no aprimoramento no processo de validação regional de tecnologias, na retroalimentação para a pesquisa e na diminuição do tempo para adoção, pelos produtores, de novas recomendações da pesquisa.

2. O T\&V propiciou uma evolução da equipe técnica, que resultou em maior eficiência na transferência de tecnologia e na assessoria técnica aos produtores.

3. O T\&V colaborou para uma maior fidelidade dos associados junto à Cooperativa.

4. Os produtores participantes do $\mathrm{T} \& \mathrm{~V}$ tiveram melhores resultados econômicos quando comparados com o total de cooperados da COPACOL.

5. O T\&V contribuiu para a viabilização das pequenas propriedades rurais (01 a $50 \mathrm{ha}$ ).

6. O T\&V COPACOL mostrou ser um aprimoramento no processo de TT tradicionalmente utilizado pelas Instituições de Pesquisa.

\section{Referências}

ALVES, E. R. A. Que fazer antes de difundir tecnologia? Tema para discussão. Cadernos de Ciência \& Tecnologia, Brasília, v. 18, n. 2, p. 135-138, maio/ago. 2001.

BENOR, D.; HARRISON, J. Q.; BAXTER, M. Agricultural extension: the training and visit system. Washington: The World Bank, 1984.

DOMIT, L. A.; LIMA, D.; ADEGAS, F. S.; DALBOSCO, M.; GOMES, C.; OLIVEIRA, A. B.; CAMPANINI, S. M. S. Manual de implantação do treino e visita $(\mathrm{T} \& \mathrm{~V})$. Londrina: Embrapa Soja, 2007. (Documentos).

DOMIT, L. A.; ADEGAS, F. S. Sistema de treino e visita $(\mathrm{T} \& \mathrm{~V})$ uma alternativa para aprimorar o processo de transferência de tecnologia. In: CONGRESSO BRASILEIRO DE SOJA, 1., 1999, Londrina. Anais... Londrina: Embrapa Soja, 1999. p. 425.

DUARTE, J. A. D. Comunicação e tecnologia na cadeia produtiva da soja em MT. 2004. Dissertação - (Mestrado em Comunicação Social) - Universidade Metodista de São Paulo, São Bernardo do Campo, SP.

EMPRESA BRASILEIRA DE PESQUISA AGROPECUÁRIA - EMBRAPA. Programa de fortalecimento e modernização de processos de transferência tecnológica. Brasília: Embrapa, Sd. (Documento, 5).

GUIMARÃES, F. C. M. S. A política de incentivo à inovação, desenvolvimento econômico e política tecnológica. Parcerias Estratégicas, Brasília, n. 9, p. 121128, out. 2000.

LEMOS, C. Inovação na era do conhecimento. Parcerias Estratégicas, Brasília, n. 8, p. 157-179, maio, 2000.

VIEIRA, O. V.; OLIVEIRA, M. F.; DOMIT, L. A. Treino e visita: experiência da Embrapa Soja e da iniciativa privada na transferência de tecnologia. Cadernos de Ciência \& Tecnologia, Brasília, v. 21, n. 2, p. 265-278, maio/ago. 2004. 
